\title{
HIP synthesis of n-carbide-type nitrides Fe3W3N and Fe6W6N and their magnetic properties
}

\section{$\operatorname{AUTHOR}(\mathrm{S}):$}

Waki, T.; Terazawa, S.; Tabata, Y.; Murase, Y.; Kato, M.; Hirota, K.; Ikeda, S.; ... Sato, K.; Kindo, K.; Nakamura, $\mathrm{H}$.

\section{CITATION:}

Waki, T.... [et al]. HIP synthesis of $\eta$-carbide-type nitrides Fe3W3N and Fe6W6N and their magnetic properties. Journal of Alloys and Compounds 2011, 509(39): 9451-9455

\section{ISSUE DATE:}

2011-09

URL:

http://hdl.handle.net/2433/147241

\section{RIGHT:}

@ 2011 Elsevier B.V.; この論文は出版社版でありません。引用の際には 出版社版をご確認ご利用ください。; This is not the published version. Please cite only the published version. 


\title{
HIP synthesis of $\eta$-carbide-type nitrides $\mathrm{Fe}_{3} \mathrm{~W}_{3} \mathrm{~N}$ and $\mathrm{Fe}_{6} \mathrm{~W}_{6} \mathrm{~N}$ and their magnetic properties
}

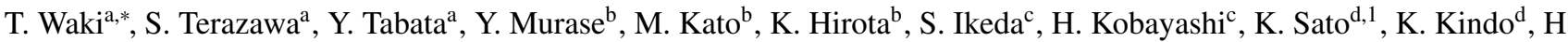 \\ Nakamura $^{\mathrm{a}}$ \\ ${ }^{a}$ Department of Materials Science and Engineering, Kyoto University, Kyoto 606-8501, Japan \\ ${ }^{b}$ Department of Molecular Chemistry and Biochemistry, Faculty of Science and Engineering, Doshisha University, Kyo-Tanabe 610-0321, Japan \\ ${ }^{c}$ Graduate School of Material Science, University of Hyogo, Kamigori, Hyogo 678-1297, Japan \\ ${ }^{d}$ The Institute for Solid State Physics, The University of Tokyo, Kashiwa 277-8581, Japan
}

\begin{abstract}
We have succeeded in synthesising iron-tungsten nitrides using the hot isostatic pressing (HIP) method and have measured their magnetic properties. Two $\eta$-carbide-type iron-tungsten nitrides with lattice constants $a=11.043(1)$ and 10.937(2) $\AA$ were synthesised directly from metal elements under high-pressure nitrogen gas. Their compositions are expected to be $\mathrm{Fe}_{3} \mathrm{~W}_{3} \mathrm{~N}$ and $\mathrm{Fe}_{6} \mathrm{~W}_{6} \mathrm{~N}$ in analogy with other $\eta$-carbide-type compounds. $\mathrm{Fe}_{3} \mathrm{~W}_{3} \mathrm{~N}$ is a ferromagnet with a Curie temperature $T_{\mathrm{C}}=110 \mathrm{~K}$ and a saturation moment $P_{\mathrm{S}}=0.78 \mu_{\mathrm{B}} / \mathrm{Fe}$, whereas $\mathrm{Fe}_{6} \mathrm{~W}_{6} \mathrm{~N}$ is an antiferromagnet with a Néel temperature $T_{\mathrm{N}}=75 \mathrm{~K}$ and shows a metamagnetic transition at around $25 \mathrm{~T}$.
\end{abstract}

Keywords: nitride materials, gas-solid reactions, sintering, magnetisation, high magnetic field, Mössbauer spectroscopy

\section{Introduction}

The magnetism of iron-based and iron-alloy-based nitrides has been studied extensively because iron tends to have a large moment and is an earth-abundant element. Several iron nitrides $\left(\alpha\right.$-FeN, $\alpha^{\prime}-\mathrm{FeN}, \alpha^{\prime \prime}-\mathrm{Fe}_{16} \mathrm{~N}_{2}, \gamma^{\prime}-\mathrm{Fe}_{4} \mathrm{~N}, \epsilon-\mathrm{Fe}_{3} \mathrm{~N}$, etc.) are known to exist [1]. Among them, $\alpha^{\prime \prime}-\mathrm{Fe}_{16} \mathrm{~N}_{2}$ has attracted much attention owing to its giant moment exceeding the value expected from the Slater-Pauling curve (for example, [2]), although several controversial reports are present $[1,2] . \mathrm{Sm}_{2} \mathrm{Fe}_{17} \mathrm{~N}_{3}$ is one of the most important iron-based nitrides because its Curie temperature, uniaxial anisotropy, and saturation moment are higher than those of $\mathrm{Nd}_{2} \mathrm{Fe}_{14} \mathrm{~B}$ (for example, [3]). In materials such as iron nitrides mentioned above and $\mathrm{Sm}_{2} \mathrm{Fe}_{17} \mathrm{~N}_{3}$, nitrogen atoms occupy interstitial sites and expand the lattice, stabilising the magnetic moment and improving the magnetic properties of these materials. However, although these materials are magnetically interesting, they cannot be used for industrial applications; this is because, these materials have low decomposition temperatures, and hence, they cannot be sintered in bulk, an important requirement for industrial use. On the other hand, ironbased nitrides containing other $d$-block elements, such as those of the $\eta$-carbide-type $\left(\mathrm{Fe}_{3} \mathrm{Mo}_{3} \mathrm{~N}\right.$ [4], $\mathrm{Fe}_{3} \mathrm{Nb}_{3} \mathrm{~N}$ [5], $\mathrm{Fe}_{3} \mathrm{~W}_{3} \mathrm{~N}$ [6], and $\mathrm{Fe}_{4} \mathrm{~W}_{2} \mathrm{~N}$ [7]) and of the filled $\beta$-Mn-type $\left(\mathrm{Fe}_{2-x} M_{x} \mathrm{Mo}_{3} \mathrm{~N}\right.$ $(M=\mathrm{Ni}, \mathrm{Pd}, \mathrm{Pt})[8])$, are obtained by heat treatments at relatively high temperatures $\left(\sim 1000^{\circ} \mathrm{C}\right)$. In order to sinter nitrides

\footnotetext{
${ }^{*}$ Corresponding author

Email address: takeshi.waki@kx7.ecs.kyoto-u.ac.jp (T. Waki)

${ }^{1}$ Present address: Ibaraki National College of Technology, Department of Natural Science 866 Nakane, Hitachinaka 312-8508, Japan
}

in bulk, a high-temperature reaction is likely to be more suitable. If these materials are found to be ferromagnetic at room temperature, they would be promising for industrial application. Recently, ferromagnetism has been reported in filled $\beta$ Mn-type nitrides [8]. Although their Curie temperatures are lower than room temperature (the highest Curie temperature of $225 \mathrm{~K}$ was obtained for $\mathrm{Fe}_{1.25} \mathrm{Pt}_{0.75} \mathrm{Mo}_{3} \mathrm{~N}$ ), it is expected that some related compounds will exhibit room-temperature ferromagnetism.

The general formula of $\eta$-carbide-type compounds with the cubic $\mathrm{Fe}_{3} \mathrm{~W}_{3} \mathrm{C}$-type crystal structure (space group $F d \overline{3} m$ ) is $M_{6}^{f} T_{4}^{e} T_{2}^{d} X_{m}^{c} X_{n}^{a}$, where $M=\mathrm{Nb}, \mathrm{Mo}, \mathrm{W}, \mathrm{Ta}, \cdots ; T=\mathrm{Cr}, \mathrm{Mn}$, $\mathrm{Fe}, \mathrm{Co}, \mathrm{Ni} ; X=\mathrm{C}, \mathrm{N}, \mathrm{O} ; m=0,2$; and $n=0,1$. The superscripts $f, e, d, c$, and $a$ represent Wyckoff positions. In the case of $m=2$ and $n=0$, that is, $T_{3} M_{3} X$, the structure is referred to as $\eta-6$ (the number represents the number of metal atoms per $X$ atom). Similarly, the case of $m=0$ and $n=1$, that is, $T_{6} M_{6} X$, is called $\eta$-12. The short $T-T$ distances of 2.5-2.6 $\AA$ suggest that these $\eta$-carbide-type compounds will exhibit itinerant electron magnetism. However, few physical properties of these $\eta$ carbide-type iron-based nitrides have been reported.

Recently, we conducted a detailed study on $\mathrm{Fe}_{3} \mathrm{Mo}_{3} \mathrm{~N}$ and found that this material does not order magnetically down to $0.5 \mathrm{~K}$ but shows a non-Fermi liquid behaviour in a low temperature region [9]. This material is located in the vicinity of the ferromagnetic quantum critical point. Ferromagnetism is easily induced by external perturbations. A very sharp itinerant electron metamagnetic transition was observed at $14 \mathrm{~T}$ at low temperatures. The substitution of a small amount of Co induces ferromagnetism with Curie temperatures lower than $20 \mathrm{~K}$ [10]. Hence, the magnetism of related iron-nitrides is also expected 
to be interesting.

In general, the difficult synthesis of nitrides prevents us from studying the physical properties of these materials. $\mathrm{Fe}_{3} \mathrm{Mo}_{3} \mathrm{~N}$, $\mathrm{Fe}_{3} \mathrm{~W}_{3} \mathrm{~N}$, and $\mathrm{Fe}_{4} \mathrm{~W}_{2} \mathrm{~N}$ were first synthesised by the ammonolysis of precursors $\mathrm{FeMoO}_{4}$ for $\mathrm{Fe}_{3} \mathrm{Mo}_{3} \mathrm{~N}$ [4] and metal-organic compounds for $\mathrm{Fe}_{3} \mathrm{~W}_{3} \mathrm{~N}$ [6] and $\mathrm{Fe}_{4} \mathrm{~W}_{2} \mathrm{~N}$ [7]. The handling of hazardous ammonia gas and air-sensitive metal-organic compounds requires special equipment. Prior and Battle developed an easy method for synthesising $\mathrm{Fe}_{3} \mathrm{Mo}_{3} \mathrm{~N}$ in a single step by heating a mixture of $\mathrm{Fe}_{2} \mathrm{O}_{3}$ and $\mathrm{MoO}_{3}$ in the proper molar ratio in a $\mathrm{N}_{2}(90 \%)-\mathrm{H}_{2}(10 \%)$ gas stream [11]. We adopted this method to synthesise $\mathrm{Fe}_{3} \mathrm{Mo}_{3} \mathrm{~N}$ in our studies on its physical properties $[9,10]$. This method also appeared to be promising for the synthesis of tungsten systems, but it was later found to be unsuitable.

A serious problem in synthesising nitrides is the desorption of nitrogen at high temperatures, resulting in the decomposition of the compound. To overcome this difficulty, we select the hot isostatic pressing (HIP) technique, which is often used to sinter nitrides such as $\mathrm{Si}_{3} \mathrm{~N}_{4}$ at high temperatures. High-pressure nitrogen gas possibly suppresses the decomposition. We found that the iron-tungsten nitrides, $\mathrm{Fe}_{3} \mathrm{~W}_{3} \mathrm{~N}$ and $\mathrm{Fe}_{6} \mathrm{~W}_{6} \mathrm{~N}$, can be synthesised directly from metal elements under high temperature and pressure. Magnetisation measurements revealed that $\mathrm{Fe}_{3} \mathrm{~W}_{3} \mathrm{~N}$ is a ferromagnet with a Curie temperature $T_{\mathrm{C}}=110$ $\mathrm{K}$ and that $\mathrm{Fe}_{6} \mathrm{~W}_{6} \mathrm{~N}$ is possibly an antiferromagnet with a Néel temperature $T_{\mathrm{N}}=75 \mathrm{~K}$.

\section{Experiments}

The HIP system (Kobe Steel, Ltd.) at Doshisha University was used in this experiment. A cold-pressed specimen, that is, a mixture of powdered iron and tungsten weighted in a proper molar ratio, was placed in a high-pressure vessel equipped with a carbon heater. The sample was capped with an alumina crucible to prevent carbon contamination. The vessel was purged several times and finally filled with nitrogen gas. The initial gas pressure was adjusted to attain the target pressure at the final temperature. The maximum temperature and pressure were $1500^{\circ} \mathrm{C}$ and $200 \mathrm{MPa}$, respectively. The temperature was raised gradually and was maintained at the target temperature for 1$2 \mathrm{~h}$. Then, the heater was turned off to cool the sample in the furnace. After the cooling, the pressure was released.

The synthesised samples were examined by X-ray diffraction (XRD) analysis (Rigaku MiniFlex) using $\mathrm{Cu} \mathrm{K} \alpha$ radiation. Electron probe microanalysis (EPMA) was performed for some samples to determine chemical compositions. The temperature dependence of the magnetisation in the range of 5 to $300 \mathrm{~K}$ and up to $7 \mathrm{~T}$ was measured using a superconducting quantum interference device (SQUID) magnetometer, MPMS (Quantum Design) at the Research Center for Low Temperature and Materials Sciences, Kyoto University. ${ }^{57} \mathrm{Fe}$-Mössbauer spectra of $\mathrm{Fe}_{3} \mathrm{~W}_{3} \mathrm{~N}$ were recorded at University of Hyogo using a constant acceleration method at several temperatures. The highfield magnetisation measurement was performed using a pulse magnet at ISSP, The University of Tokyo, up to $54 \mathrm{~T}$ at $4.2 \mathrm{~K}$.

\section{Results and Discussion}

\subsection{Synthesis and Phase Analysis}

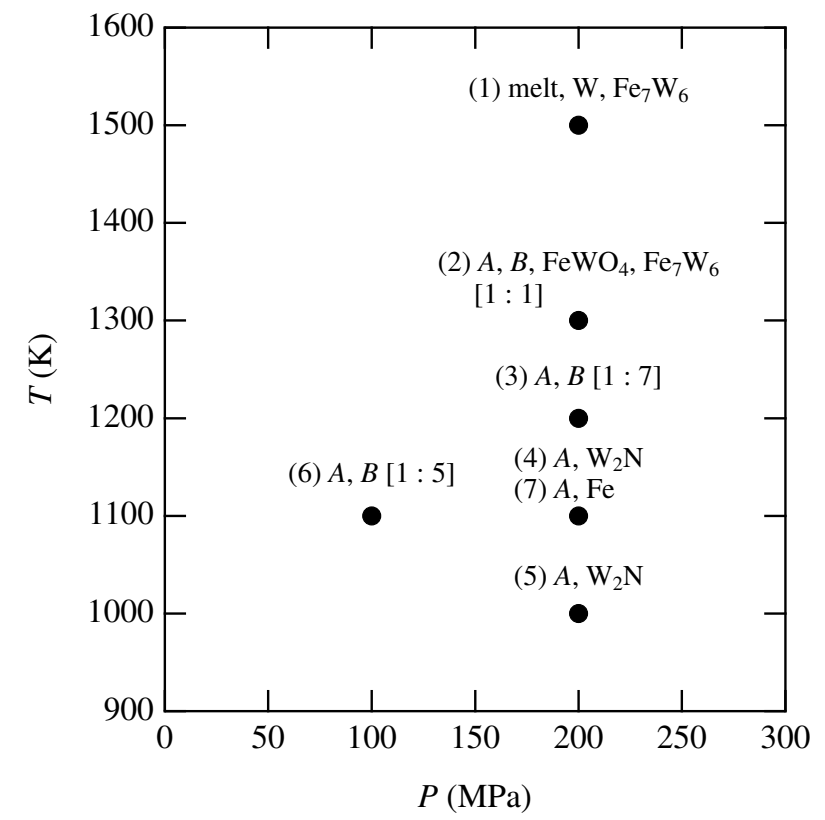

Figure 1: HIP temperature and pressure conditions and obtained phases. The numbers in parenthesis indicate the order in which the experiments were conducted. The numbers in brackets represent the ratio of $A$ to $B$ estimated from XRD main peaks. The phases $A$ and $B$ are identified as $\mathrm{Fe}_{3} \mathrm{~W}_{3} \mathrm{~N}$ and $\mathrm{Fe}_{6} \mathrm{~W}_{6} \mathrm{~N}$, respectively.

Figure 1 summarises the heat treatment conditions, namely, temperature and pressure, and the results of XRD characterisation. The numbers in parenthesis indicate the order in which the experiments were conducted. Case $1\left(1500^{\circ} \mathrm{C}, 200 \mathrm{MPa}\right)$ was studied first. Although no liquid phase exists below $1500^{\circ} \mathrm{C}$ in the Fe-W phase diagram [12], the surface of the specimen was found to be melted. XRD showed that the sample consists of $\mathrm{W}$ and $\mathrm{Fe}_{7} \mathrm{~W}_{6}$. No nitride phase was observed.

Figure 2 shows the XRD pattern of sample $2\left(1300^{\circ} \mathrm{C}\right.$, $200 \mathrm{MPa}$ ) with the calculated pattern of $\mathrm{Fe}_{3} \mathrm{~W}_{3} \mathrm{~N}$ [6]. We found two sets of peaks, which were characteristic for the $\eta$-carbide-type structure. These $\eta$-carbide-type iron-tungstennitrides were synthesised from pure elements for the first time. We name the phase with the larger lattice constant $A$ (corresponding to the reflections indicated by solid triangles) and that with the smaller lattice constant $B$ (open triangles). The lattice constants are estimated to be 11.043(1) and 10.937(2) $\AA$ for $A$ and $B$, respectively. The lattice constants of compounds with the $\eta-6$ structure are generally larger than those of compounds with the $\eta-12$ structure by about $1 \%$ when the compounds are composed of the same elements. For example, in the case of the Fe-W-C system, lattice constants of $11.10-11.14 \AA$ and 10.96 $\AA$ have been reported for $\eta-6$ and $\eta$-12, respectively [13, 14]. Because the lattice constant of $A$ is larger than that of $B$ by $0.96 \%$, it is reasonable to consider that $A$ and $B$ have $\eta-6$ and 


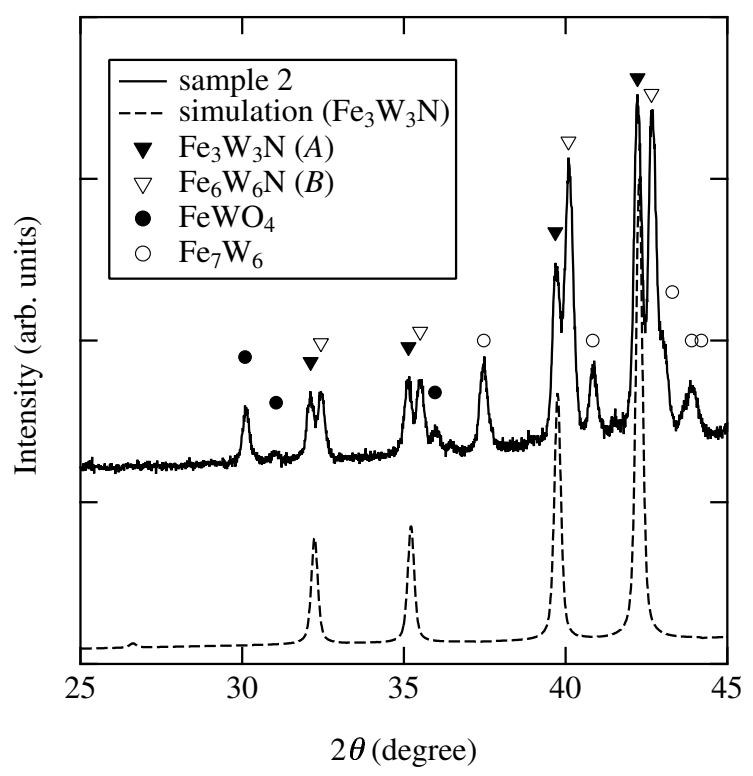

Figure 2: The XRD pattern of sample 2 (solid curve). The simulated pattern of $\mathrm{Fe}_{3} \mathrm{~W}_{3} \mathrm{~N}[6]$ is represented by the dashed curve. Solid triangles, open triangles, solid circles, and open circles indicate reflections of $\mathrm{Fe}_{3} \mathrm{~W}_{3} \mathrm{~N}$ ( $A$ in the text), $\mathrm{Fe}_{6} \mathrm{~W}_{6} \mathrm{~N}(B), \mathrm{FeWO}_{4}$, and $\mathrm{Fe}_{7} \mathrm{~W}_{6}$, respectively.

$\eta-12$ structures, respectively. In the Fe-W-C system, solid solutions between $\mathrm{Fe}_{2} \mathrm{~W}_{4} \mathrm{C}$ and $\mathrm{Fe}_{4} \mathrm{~W}_{2} \mathrm{C}$ are known to exist as $\eta$ - 6 [13]. The presence of both $\mathrm{Fe}_{3} \mathrm{~W}_{3} \mathrm{~N}$ and $\mathrm{Fe}_{4} \mathrm{~W}_{2} \mathrm{~N}[6,7]$ suggests the possible formation of solid solutions between them, analogous with the case of carbides, but $A$ will be reasonably identified as $\mathrm{Fe}_{3} \mathrm{~W}_{3} \mathrm{~N}$ from EPMA, as mentioned below. To the best our knowledge, $B$ is a $\eta-12$ iron-tungsten nitride, synthesised for the first time. The remaining peaks are assigned to either $\mathrm{FeWO}_{4}$ or $\mathrm{Fe}_{7} \mathrm{~W}_{6}$. The origin of oxygen included in $\mathrm{FeWO}_{4}$ is unclear. Surface oxidation of the metal powder, oxygen impurity in the nitrogen gas, and residual air in the pressure vessel are possible sources. To reduce the oxide impurity, after case 2, we attempted the following procedure: (1) a coldpressed sample was heated in a hydrogen gas stream at $1000{ }^{\circ} \mathrm{C}$ for $48 \mathrm{~h}$ to reduce its iron oxide content before the HIP treatment, (2) pieces of Ti ingots were placed in the pressure vessel as an oxygen getter, and (3) the surface of the obtained sample was etched to remove the oxide layer. As a result, the $\mathrm{FeWO}_{4}$ content was reduced considerably. According to the phase diagram [12], $\mathrm{Fe}_{7} \mathrm{~W}_{6}$ is stable between 1215 and $1637^{\circ} \mathrm{C}$ when $\mathrm{Fe}$ : $\mathrm{W}$ is approximately $1: 1$; therefore, we limited the temperature of the heat treatment to below $1200^{\circ} \mathrm{C}$ after case 2 .

In cases 2,3 and $6, A$ and $B$ were found to coexist, while $B$ did not appear in cases 4 and 5. $A$ seems to be stable at $P \geq 200 \mathrm{MPa}$ and $T \leq 1100^{\circ} \mathrm{C}$. On the other hand, $B$ is stable at high temperatures of $T \geq 1200^{\circ} \mathrm{C}$ when $P=200 \mathrm{MPa}$ and $T \geq 1100^{\circ} \mathrm{C}$ when $P=100 \mathrm{MPa}$. The composition of the products was found to be insensitive to the holding time if the reaction temperature is the same, indicating that all the reaction was already completed in the shortest period of the heat treatments. It is likely that the $A$ observed in cases 2,3 , and 6 ap- peared during the cooling process. To obtain $B$ in a pure form, rapid quenching from the reaction temperature may be necessary. Unfortunately, we have not yet succeeded in synthesising $B$ in a single phase.

From the above examination, we concluded that a temperature below $1100{ }^{\circ} \mathrm{C}$ and a pressure of $200 \mathrm{MPa}$ are the appropriate conditions for obtaining a single phase of $A$. The appearance of $\mathrm{W}_{2} \mathrm{~N}$ seems to be due to the deviation of the iron-tungsten ratio from the nominal composition. To prevent the appearance of $\mathrm{W}_{2} \mathrm{~N}$, we added excess iron to a sample and heated it to $1100^{\circ} \mathrm{C}$, under $200 \mathrm{MPa}$ (sample 7). The XRD pattern of sample 7 is shown in Fig. 3. $A$ and Fe were observed (dashed curve). The product was ferromagnetic at room temperature because of the inclusion of the Fe impurity. It was soaked in dilute hydrochloric acid for two days to remove the iron. The solid curve represents the XRD pattern of the product after the treatment, in which the amount of ferromagnetic Fe was markedly reduced. Thus, we successfully obtained a fairly purified single phase of the $\eta-6 \mathrm{Fe}-\mathrm{W}-\mathrm{N}$ phase. Using EPMA

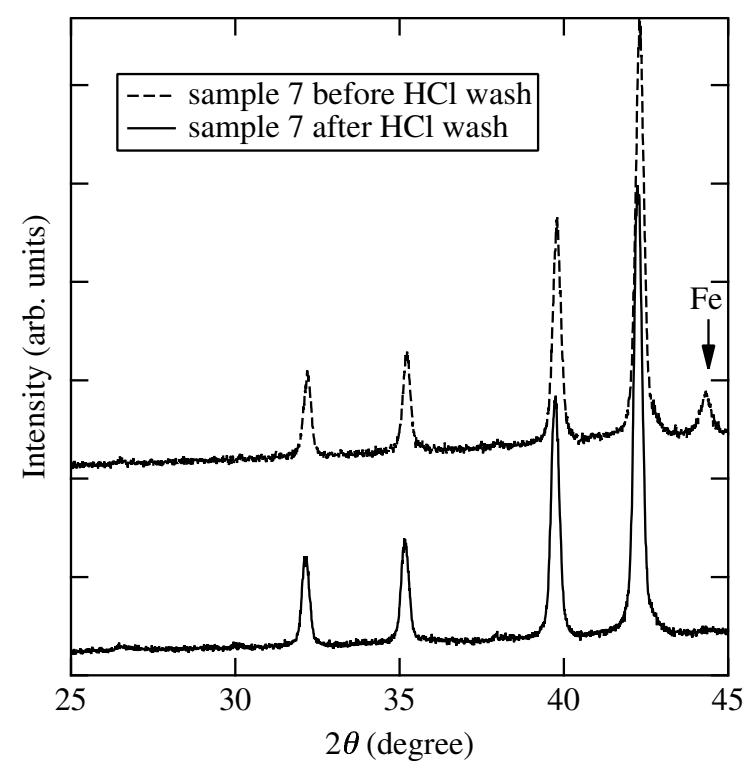

Figure 3: XRD patterns of sample 7 before and after $\mathrm{HCl}$ washing. We obtained almost pure $\mathrm{Fe}_{3} \mathrm{~W}_{3} \mathrm{~N}$ after the successful removal of the $\mathrm{Fe}$ impurity.

(Fe- $K_{\alpha}$ and W- $M_{\alpha}$ emissions), we estimated the chemical composition of metal elements in the $\mathrm{HCl}$-washed specimen to be $\mathrm{Fe} / \mathrm{W}=1.09(4)$. Thus, it is reasonable to assign $A$ to $\mathrm{Fe}_{3} \mathrm{~W}_{3} \mathrm{~N}$.

On the other hand, it was not easy to determine the composition of the impurity-containing $B$ phase. In $\eta$ - 12 carbides, however, it is known that $3 d$ and $(4 d, 5 d)$ atoms rarely replace each other $[13,14,15]$. In what follows, we regard $A$ and $B$ as $\mathrm{Fe}_{3} \mathrm{~W}_{3} \mathrm{~N}$ and $\mathrm{Fe}_{6} \mathrm{~W}_{6} \mathrm{~N}$, respectively.

We applied the same method to synthesise $\mathrm{Fe}_{3} \mathrm{Nb}_{3} \mathrm{~N}$, but in vain.

\subsection{Magnetic properties}

Figure 4(a) shows the magnetisation of $\mathrm{Fe}_{3} \mathrm{~W}_{3} \mathrm{~N}$ measured for the sample 7 after the excess $\mathrm{Fe}$ was removed. The mag- 
netisation was normalised with respect to the chemical formula $\mathrm{Fe}_{3} \mathrm{~W}_{3} \mathrm{~N}$. Even at room temperature, small spontaneous magnetisation was observed. However, at high fields, the magnetisation increases almost linearly. Between 150 and $300 \mathrm{~K}$, the spontaneous magnetisation $M_{0}$ estimated by extrapolating the magnetisation at high fields to $0 \mathrm{~T}$ is constant, whereas the slope above $1 \mathrm{~T}$ depends on the temperature. This offset is ascribed to ferromagnetic impurities with high Curie temperatures, most probably residual iron. Below $110 \mathrm{~K}$, the spontaneous magnetisation exceeds $M_{0}$. This component corresponds to the intrinsic ferromagnetism of $\mathrm{Fe}_{3} \mathrm{~W}_{3} \mathrm{~N}$. At the lowest temperature, the magnetisation is saturated to $2.9 \mu_{\mathrm{B}}$ per formula unit (f.u.). Subtracting the impurity contribution, the average moment per Fe is estimated to be $P_{\mathrm{S}}=0.78 \mu_{\mathrm{B}} / \mathrm{Fe}$. This is much smaller than that of $\alpha$-Fe $\left(2.22 \mu_{\mathrm{B}} / \mathrm{Fe}\right)$.

In Fig. 4(b), we show the temperature dependence of $M / H$, where $M$ was measured at a field of $0.1 \mathrm{~T}$. The contribution of the ferromagnetic impurity was subtracted assuming that the impurity component is independent of temperature. Furthermore, the absolute values of $M / H$ were corrected assuming that the value at $300 \mathrm{~K}$ coincides with the high-field susceptibility at the same temperature. At high temperatures, the reciprocal susceptibility is linear, obeying the Curie-Weiss (CW) law. By fitting the data above $130 \mathrm{~K}$ with the $\mathrm{CW}$ function, we obtained a Weiss temperature $\theta=110 \mathrm{~K}$ and an effective moment $P_{\text {eff }}=3.4 \mu_{\mathrm{B}} / \mathrm{Fe}$. With decreasing temperature, $M / H$ shows a rapid increase below $110 \mathrm{~K}$ and saturates at low temperatures, leading to the conclusion that $\mathrm{Fe}_{3} \mathrm{~W}_{3} \mathrm{~N}$ is a ferromagnet with a Curie temperature $T_{\mathrm{C}}=110 \mathrm{~K}$. The ratio $P_{\text {eff }} / P_{\mathrm{S}}=4.3$ is much larger than unity. This is characteristic for a weak itinerant electron ferromagnet.

Figure 5 shows Mössbauer spectra measured at different temperatures. At $300 \mathrm{~K}$, a dominant singlet was observed, indicating no magnetic order at this temperature. On the right side of the main absorption, a small dip was observed, which may originate from a paramagnetic impurity phase. At 77 and $4.6 \mathrm{~K}$, the spectrum was broadened, indicating the appearance of a relatively small internal field at these low temperatures. This is consistent with the ferromagnetic order below $T_{\mathrm{C}}=110 \mathrm{~K}$ and the small ordered moment $\left(0.78 \mu_{\mathrm{B}} / \mathrm{Fe}\right)$ revealed from the magnetisation measurement. The origin of the somewhat complicated structure of the spectrum at the lowest temperature is unclear, but it may be related to either the presence of two crystallographically inequivalent $\mathrm{Fe}$ sites in the $\eta$-carbide-type structure ( $16 d$ and $32 e$ ) or the inclusion of the impurity phase.

Although we have not succeeded in synthesising $\mathrm{Fe}_{6} \mathrm{~W}_{6} \mathrm{~N}$ in a pure form, we performed magnetisation measurements on sample 3 to determine the qualitative magnetic behaviour of $\mathrm{Fe}_{6} \mathrm{~W}_{6} \mathrm{~N}$. The magnetisation was normalised with respect to the chemical formula of $\mathrm{Fe}_{6} \mathrm{~W}_{6} \mathrm{~N}$. The possible contribution of ferromagnetic $\mathrm{Fe}_{3} \mathrm{~W}_{3} \mathrm{~N}$ may induce a large error. Figure 6 shows the temperature dependence of $M / H$ measured under a relatively high field of $5.5 \mathrm{~T}$ to reduce the contribution of ferromagnetic impurities. $M / H$ shows a $\mathrm{CW}$-type temperature dependence at high temperatures and a clear cusp at $75 \mathrm{~K}$, suggesting the onset of antiferromagnetic order.

Figure 7 shows the result of the high-field magnetisation
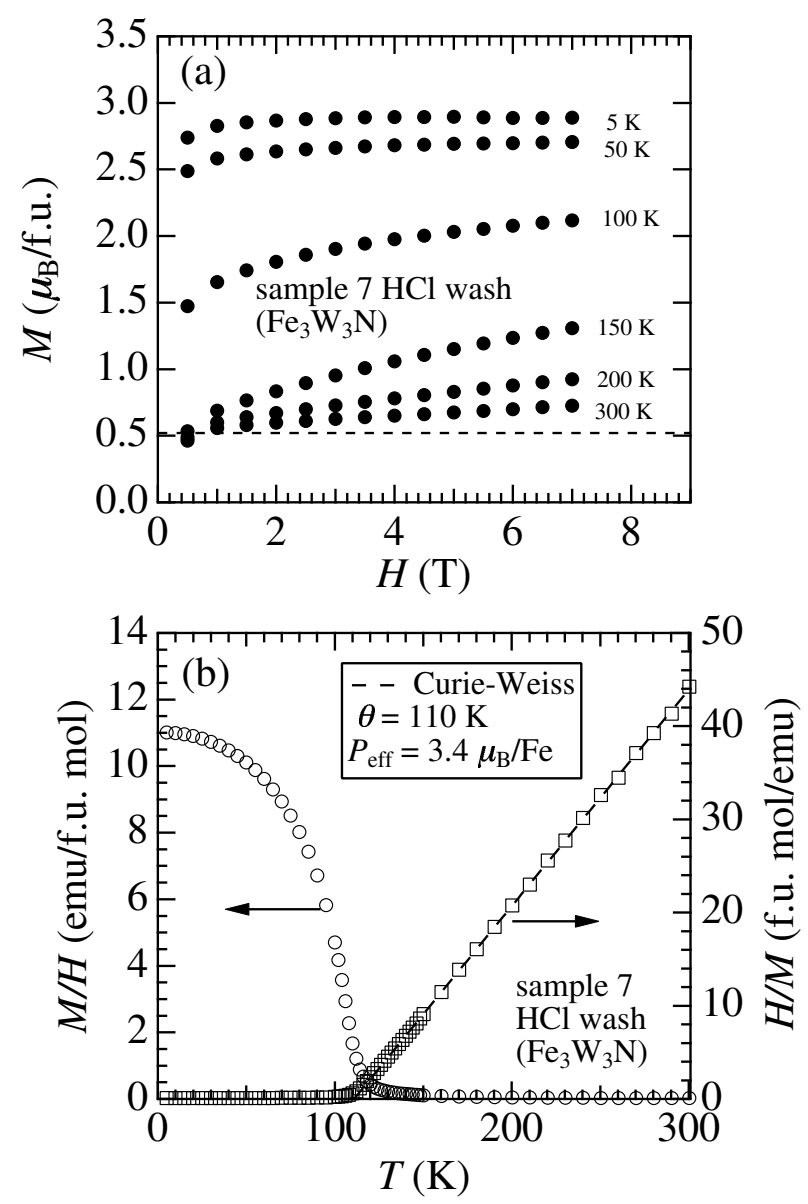

Figure 4: (a) Magnetisation curves of $\mathrm{Fe}_{3} \mathrm{~W}_{3} \mathrm{~N}$ (sample 7 after $\mathrm{HCl}$ wash) at several temperatures. At high temperatures, the magnetisation increases rapidly at low fields and then linearly at high fields. This rapid increase is ascribed to ferromagnetic impurities. The dashed line is the base line of intrinsic $\mathrm{Fe}_{3} \mathrm{~W}_{3} \mathrm{~N}$ estimated by extrapolating high-field magnetisation above $T_{\mathrm{C}}$. Subtracting the impurity magnetisation, the intrinsic saturation moment at the lowest temperature is estimated to be $P_{\mathrm{s}}=0.78 \mu_{\mathrm{B}} / \mathrm{Fe}$. (b) Temperature dependences of corrected $M / H$ (left axis) and its reciprocal (right axis). The dashed line represents the best fit with the Curie-Weiss law with $P_{\text {eff }}=3.4 \mu_{\mathrm{B}} / \mathrm{Fe}$ and $\theta=110 \mathrm{~K}$. 


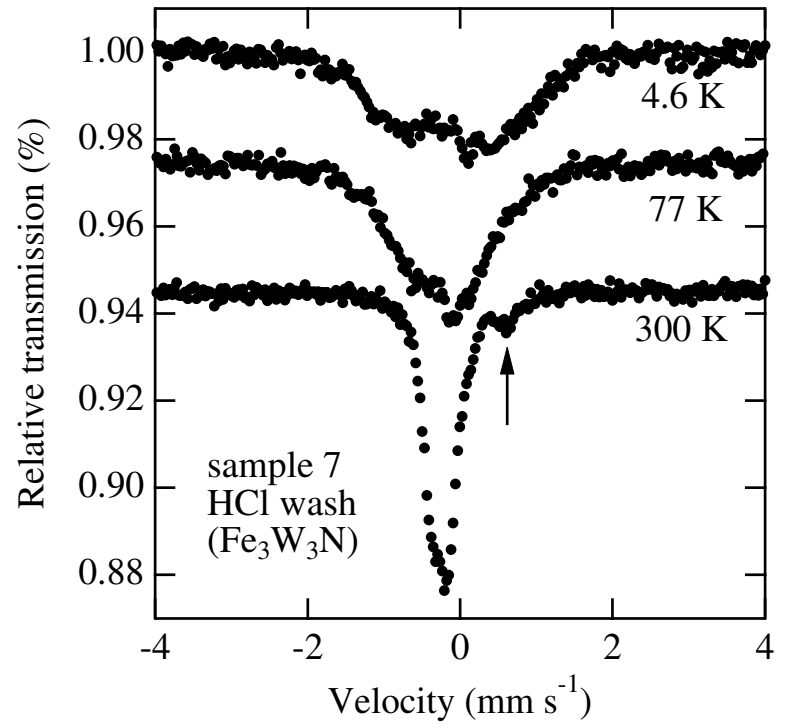

Figure 5: Mössbauer spectra of $\mathrm{Fe}_{3} \mathrm{~W}_{3} \mathrm{~N}$ (sample 7 after $\mathrm{HCl}$ wash) measured at 4.6, 77, and $300 \mathrm{~K}$. At $300 \mathrm{~K}$, a main absorption singlet and a small dip (arrow) ascribed to an impurity phase were observed. With decreasing temperature, the spectrum is broadened.

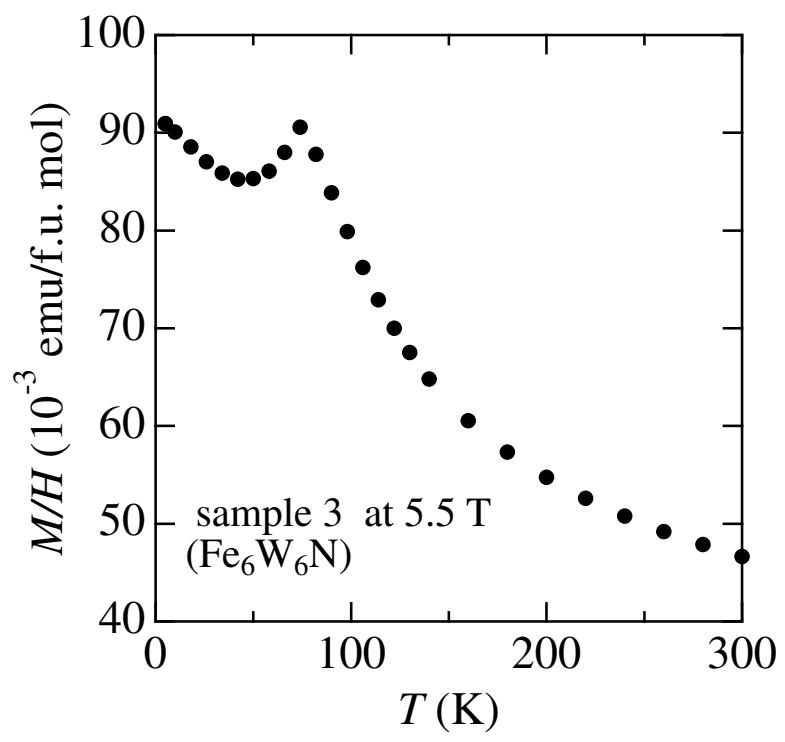

Figure 6: The temperature dependence of $M / H$ for $\mathrm{Fe}_{6} \mathrm{~W}_{6} \mathrm{~N}$ (sample 3) measured at $5.5 \mathrm{~T}$. $M / H$ is normalised per $\mathrm{Fe}_{6} \mathrm{~W}_{6} \mathrm{~N}$ formula unit. At high temperatures, it shows a CW-type behaviour. A sharp peak is present at $75 \mathrm{~K}$. measurement for $\mathrm{Fe}_{6} \mathrm{~W}_{6} \mathrm{~N}$ at $4.2 \mathrm{~K}$. The small spontaneous magnetisation is ascribed to ferromagnetic impurities. The magnetisation curve between 1 and $20 \mathrm{~T}$ is nearly linear. A jump with large hysteresis was observed at around $25 \mathrm{~T}$. The metamagnetic transition is most probably due to spin flip in the antiferromagnetic state. As seen in $d M / d H$, two anomalies were observed at the metamagnetic transition. This would be related to the presence of two crystallographically inequivalent Fe sites $(16 d$ and $32 e)$.

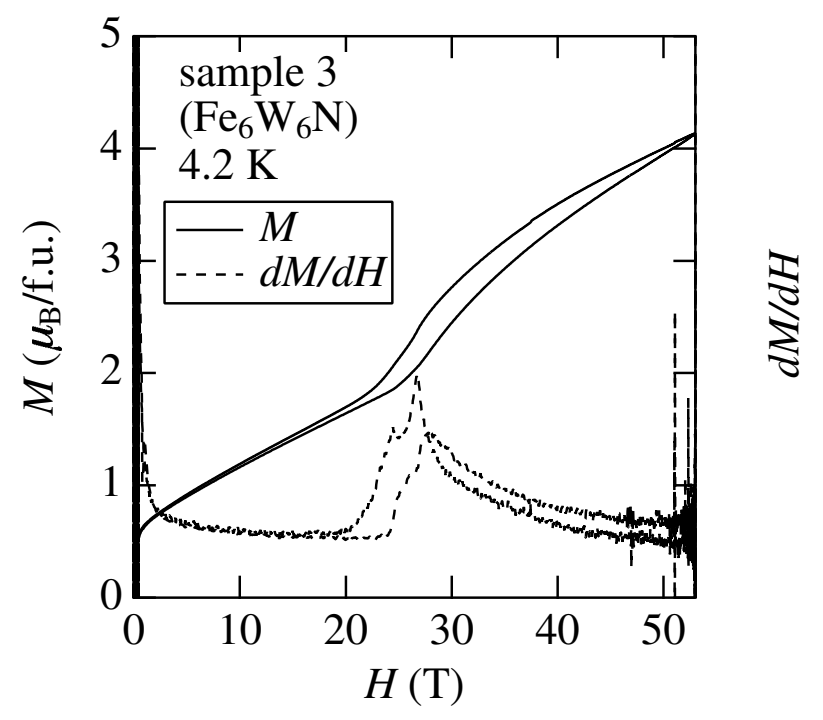

Figure 7: The high-field magnetisation of $\mathrm{Fe}_{6} \mathrm{~W}_{6} \mathrm{~N}$ (sample 3) (left axis). $d M / d H$ vs. $H$ (right axis). Around $25 \mathrm{~T}$, two anomalies were observed in $d M / d H$.

\section{Conclusions}

We successfully synthesised the $\eta$-carbide-type iron-tungsten nitrides $\mathrm{Fe}_{3} \mathrm{~W}_{3} \mathrm{~N}$ and $\mathrm{Fe}_{6} \mathrm{~W}_{6} \mathrm{~N}$ from mixtures of iron and tungsten powder by heating them under high-pressure nitrogen gas using the HIP technique. $\mathrm{Fe}_{3} \mathrm{~W}_{3} \mathrm{~N}$ is ferromagnetic with $T_{\mathrm{C}}=110 \mathrm{~K}$ and $P_{\mathrm{S}}=0.78 \mu_{\mathrm{B}} / \mathrm{Fe} . \quad \mathrm{Fe}_{6} \mathrm{~W}_{6} \mathrm{~N}$ is a newly synthesised $\eta$-12 type nitride and shows antiferromagnetic order at $T_{\mathrm{N}}=75 \mathrm{~K}$. Interestingly, the magnetism of Fe-W-N $\eta$-carbides was found to be sensitive to the site occupied by nitrogen, in contrast to the case of Co-Mo-N $\eta$-carbides [16]. Systematic studies on related compounds must be curried out to clarify the role of nitrogen in $\eta$-carbide-type nitrides.

\section{Acknowledgements}

This study was supported by a grant from Kinki Regional Invention Center (foundation), a Grant-in-Aid for Scientific Research on Priority Areas 'Novel States of Matter Induced by Frustration' (19052003), a Grant-in-Aid for the Global COE Program, 'International Center for Integrated Research and Advanced Education in Materials Science', and a Grant-in-Aid for Young Scientists (B) (21760531) from the Ministry of Education, Culture, Sports, Science and Technology of Japan. 


\section{References}

[1] J. M. D. Coey, P. A. I. Smith, J. Magn. Magn. Mater. 200 (1999) 405.

[2] M. Takahashi, H. Shoji, J. Magn. Mang. Mater. 208 (2000) 145.

[3] Y. Otani, D. P. F. Hurley, H. Sun, J. M. D. Coey, J. Appl. Phys. 69 (1991) 5584.

[4] D. S. Bem, C. P. Gibson, H.-C. zur Loye, Chem. Mater. 5 (1993) 397.

[5] F. X. Lü, K. H. Jack, J. Less-Common Met. 14 (1985) 123.

[6] K. S. Weil, P. N. Kumta, Acta Cryst. C53 (1997) 1745.

[7] K. S. Weil, P. N. Kumta, J. Solid State Chem. 134 (1997) 302.

[8] T. J. Prior, S. E. Oldham, V. J. Couper, P. D. Battle, Chem. Mater. 17 (2005) 1867.

[9] T. Waki, S. Terazawa, Y. Tabata, F. Oba, C. Michioka, K. Yoshimura, S. Ikeda, H. Kobayashi, K. Ohoyama, H. Nakamura, J. Phys. Soc. Jpn. 79 (2010) 043701.

[10] T. Waki, S. Terazawa, T. Yamazaki, Y. Tabata, K. Sato, A. Kondo, K. Kindo, M. Yokoyama, Y. Takahashi, H. Nakamura, EPL 94 (2011) 37004.

[11] T. J. Prior, P. D. Battle, J. Solid State Chem. 172 (2003) 128.

[12] S. V. N. Naidu, A. M. Sriramamurthy, P. R. Rao, J. Alloy Phase Diagrams 2 (1986) 176

[13] C. B. Pollock, H. H. Stadelmaier, Metall. Trans. 1 (1970) 767.

[14] P. Ettmayer, R. Suchentrunk, Monatsh. Chem. 101 (1970) 1098.

[15] A. C. Fraker, H. H. Stadelmaier, Trans. TMS-AIME 245 (1969) 847.

[16] S. M. Hunter, D. Mckay, R. I. Smith, J. S. J. Hargreaves, D. H. Gregory, Chem. Mater. 22 (2010) 2898. 